\title{
Análisis del patrón de carrera sobre superficie artificial y natural en futbolistas adolescentes Analysis of the running pattern on artificial and natural surface in adolescent football players \\ **Brandon López-Gómez, , **David Andrés Pérez-Mendoza, , **Julián Santiago Guzmán-Revelo, **Luis Gabriel Rangel- Caballero, *Yully Corzo-Vargas, ***Tábata de Paula Facioli., *Adriana Angarita-Fonseca, ***Juan Carlos Sánchez- Delgado \\ *Universidad de Santander (Colombia), ** Universidad Santo Tomás, (Colombia), *** Universidad de São Paulo (Brasil)
}

Resumen. Introducción: Existe poca evidencia que detalle el comportamiento de cada variable espacio-temporal del patrón de carrera utilizando diferentes superficies. Objetivo: Comparar las variables espaciotemporales del patrón de carrera de futbolistas adolescentes en superficie natural y artificial. Método: se realizó un estudio de corte transversal con 18 jugadores de fútbol masculino (edad mediana = 12 años; Rango intercuartílico [RIC] 12-13). Mediante un sistema de medición óptico de 5 metros de longitud se analizó velocidad, aceleración, tiempo de contacto (Tc), tiempo de vuelo (Tv), fase de contacto, fase de apoyo, propulsión, zancada y cadencia. Las valoraciones fueron inicialmente desarrolladas en Superficie Artificial (SA) y 24 horas después en Superficie Natural (SN). Se utilizaron las pruebas Rangos con signos de Wilcoxon para datos pareados y el coeficiente de correlación de Spearman. Resultados: La SA mostró una fase de apoyo superior a la SN (SN: Me=0,05 RIC:0,03; 0,06; SA: Me=0,09 RIC 0,08;0,10; $p<0,001$ ). El Tv (SN: Me=0,16 RIC:0,14;0,19; SA: Me=0,04 RIC: 0,04;0,05; $\mathrm{p}<0,001$ ), la fase de contacto (SN: Me=0,02 RIC:0,02;0,03; SA: Me=0,02 RIC: 0,01;0,02; $\mathrm{p}=0,040$ ) y la propulsión (SN: Me=0,14 RIC:0,09;0,17; SA: Me=0,07 RIC:0,06;0,09; $\mathrm{p}=<0,001$ ) fueron mayores en SN que en SA. Se encontró una relación indirecta entre velocidad y fase de contacto en SN. El Tv y la zancada se asociaron indirectamente con la aceleración en SA. Conclusión: el patrón de carrera varía según la superficie utilizada. La fase de contacto puede explicar la velocidad en la SN; mientras que el Tv y la zancada pueden explicar la aceleración en la SA.

Palabras Claves: Fútbol; carrera; locomoción; aceleración; cinética.

\begin{abstract}
Introduction: There is little evidence that details the behavior of each spatio-temporal variable of the running pattern using different surfaces. Objective: To compare the spatio-temporal variables of the running pattern over two surfaces in adolescent soccer players. Method: A cross-sectional study involving 18 male soccer players was conducted (median $[\mathrm{Me}]$ age $=12$ years; Interquartile range [IQR] 12-13). Speed, acceleration, contact time (Ct), flight time (Ft), contact phase, support phase, propulsion, stride, and cadence were evaluated through a 5-meter long optical measurement system. The assessments were initially carried out in an Artificial Surface (AS) and 24 hours later in a Natural Surface (NS). The Wilcoxon signed-rank test for paired data and the Spearman correlation coefficient were used. Results: The support phase was greater in AS than NS (NS: Me $=0.05$ IQR: 0.03; 0.06; AS: Me $=0.09$ IQR 0.08; 0.10; p $<0.001$ ). The Ft (NS: Me =0.16 IQR: 0.14; 0.19; AS: Me=0.04 IQR: 0.04; 0.05; $<<0.001$ ), the contact phase (NS: Me=0.02 IQR: 0.02 ; 0.03; AS: $\mathrm{Me}=0.02$ IQR: 0.01; 0.02; $\mathrm{p}=0.040$ ) and propulsion (NS: Me = 0.14 IQR: 0.09; 0.17; AS: Me = 0.07 IQR: 0.06; 0.09; p= $<0.001)$ were greater in NS than AS. An indirect relationship between speed and contact phase in NS was found. The Ft and the stride were indirectly associated with acceleration in AS. Conclusion: The running pattern varies according to the surface used. The contact phase can explain the speed in the NS; while the Ft and the stride can explain the acceleration in AS.
\end{abstract}

Key Word: Soccer; running; locomotion; acceleration; kinetics.

\section{Introducción}

Las habilidades de carrera han adquirido gran relevancia en el fútbol. Lo anterior se evidencia en que los jugadores son cada día más rápidos y que el sprint en línea recta es la acción que con mayor frecuencia se desarrolla antes del gol (Faude, Koch \& Meyer, 2012). Si bien, los aspectos técnicos, tácticos y físicos han sido ampliamente estudiados y considerados como factores determinantes del éxito en el futbolista, existen otros relacionados con la biomecánica de la carrera que siguen sin estar claros. (Haugen, Tønnessen, Hisdal \& Seiler, 2014; Murata, Takai, Kanehisa, Fukunaga \& Nagahara, 2018; Oyarzo-Mauricio, Said-Negrete \& NazarAraya, 2017; Sánchez, Hernández, Muñoz, Gonzales, Fernández \& Carretero, 2016).

El comportamiento de las variables espaciotemporales, principalmente la velocidad y aceleración, son determinantes de la habilidad de carrera en el futbolista (Morin et al., 2015; Murata et al., 2018; Nagahara, Mizutani, Matsuo, Kanehisa \& Fukunaga, 2018; Rabita, Dorel, Slawinski, Saez-

Fecha recepción: 29-04-19. Fecha de aceptación: 04-12-19 Juan Carlos Sanchez Delgado ju.sanchez@mail.udes.edu.co
de-Villarreal, Couturier, Samozino \& Morin, 2015; González de los Reyes, Fernández-Ortega \& Garavito-Peña, 2019). En este punto, la literatura científica que relaciona la velocidad o la aceleración con las demás variables espaciotemporales, no es concluyente. Según Murphy, Lockie \& Coutts, (2003) y Wild, Bezodis, North \& Bezodis, (2018) los sujetos más rápidos son aquellos que poseen una mayor frecuencia de pasos durante la fase de aceleración inicial, contrario a lo referido por Murata et al., 2018, quienes indican que las mayores velocidades de carrera se logran con una mayor longitud de paso durante la misma fase de aceleración.

Además de las variables espaciotemporales, las fuerzas de reacción determinadas por el tipo de suelo donde se juega también pueden influir en la habilidad de carrera (Williams, Akogyrem, \& Williams, 2013; Sánchez-Sánchez-Sánchez, García-Unanue, Jimenez-Reyes, Viejo-Romero \& GómezLópez, 2016; Ávalos-Guillén et al., 2017, Gallardo, Felipe, Burillo \& Gallardo, 2010, Zanetti, Bigardi, Franceschini \& Audenio, 2013). Específicamente, Gaudino, Gaudino, Alberti, \& Minetti (2013) mostraron que, en superficie natural y artificial, la velocidad y la aceleración son superiores al ser comparadas con estas mismas en superficie de arena. Otro estudio, el de Andersson, Ekblom, \& Krustrup (2008), no encontró diferencias en los patrones de movimiento realizados entre 
la SA y SN. Por otra parte, Nédélec, McCall, Carling, Gall, Berthoin \& Dupont (2013) y Gain, Swedenhjelm, Mayhew, \& Houser (2010) describieron una mayor velocidad de carrera en SA, comparada con la SN, lo cual guarda coincidencia con los resultados obtenidos por Choi, Raymond, \& Elean (2015).

Teniendo en cuenta lo anterior, se hace necesario analizar el comportamiento de las variables espaciotemporales del patrón de carrera en SN y SA, lo cual permitirá comprender mejor los posibles determinantes de un mejor rendimiento en la carrera y sugerir modalidades de entrenamiento en los futbolistas juveniles.

\section{Material y método}

Se realizó una investigación de corte trasversal, en 18 sujetos de sexo masculino, con un promedio de edad de $12,4 \pm 0,5$ años, quienes pertenecían a la Selección Infantil de Fútbol de Santander (SIFS). Todos participaron con previa lectura y firma de asentimiento y consentimiento informado. Fueron excluidos aquellos participantes que presentaron algún tipo de lesión musculoesquelética en los últimos tres meses $(n=1)$ y aquellos que no completaron el proceso de evaluación $(\mathrm{n}=3)$.

El comité de investigación del programa de Cultura Física, Deporte y Recreación de la Universidad Santo Tomás Seccional Bucaramanga y el de Fisioterapia de la Universidad de Santander, aprobaron la realización del presente estudio en el acta $\mathrm{N}^{\circ} 9$ del 2017, para formalizar el convenio de cooperación interinstitucional para su desarrollo (B-283).

Dentro de las variables evaluadas se encuentran el género, la edad, la posición de juego, la talla medida con estadiómetro (SECA 213, Alemania) graduado en centímetros $(\mathrm{cm})$ y milímetros $(\mathrm{mm})$, y se leyó con una precisión de $0.1 \mathrm{~cm}$; se registró el peso corporal con báscula (Tanita 679, Japón) con una precisión de 100 gramos, para finalmente calcular el índice de masa corporal (IMC). Las variables espaciotemporales evaluadas fueron: a) la fase de contacto, definida como el tiempo desde el primer toque del talón con la superficie hasta el apoyo total del pie; b) la fase de apoyo, considerada el periodo en que el pie permanece completamente en contacto con el suelo; c) la fase de propulsión, que corresponde al tiempo desde el ascenso del talón hasta el levantamiento de la punta del pie; d) la zancada, definida como la distancia entre las puntas de las huellas sucesivas del mismo pie; e) la cadencia, que se entiende como el ritmo expresado en pasos por segundo; f) la velocidad, considerada como la relación entre la distancia de los dos pies y la suma del primer Tiempo de Contacto (Tc) y el Tiempo de Vuelo (Tv). g) Por último, se evaluó la aceleración que es la relación entre el delta de las velocidades de los dos pasos y la suma de los tiempos de contacto y de vuelo (Optogait, 2018; Blazebich, 2011)

Para el análisis de las variables espaciotemporales se utilizó un sistema de medición óptico (Optogait, Italia) formado por cinco barras transmisoras y cinco receptoras; cada una mide un 1 metro de largo y contiene 96 sensores ópticos que detectan las interrupciones de comunicación causadas por el movimiento del sujeto evaluado; el sistema permite que los tiempos de contacto y de vuelo puedan ser medidos con una frecuencia de $1 \mathrm{KHz}$ y con una precisión de $1 \mathrm{~cm}$ (Optogait, 2018). Este dispositivo ha mostrado un Coeficiente de Correlación Intraclase mayor de 0,9 al ser comparado con otros sistemas de medición de las variables espaciotemporales (Lee, Song, Lee, Jung, Shin \& Shin, 2014; Lienhard, Schneider \& Maffiuletti, 2013).

\section{Procedimiento}

Una vez socializado los objetivos del estudio y firmados los consentimientos, se procedió a realizar la valoración antropométrica de peso, talla e IMC. Posteriormente, los sujetos fueron sometidos a un calentamiento de 10 minutos a intensidad de 3/10 Borg modificado, antes de realizar dos intentos del patrón de carrera a máxima velocidad sobre SA de monofilamento (Domo ${ }^{\circledR}$ Slide DS); finalmente, se seleccionó el mejor de los dos registros. 24 horas después se procedió a realizar el mismo proceso de evaluación del patrón de carrera sobre $\mathrm{SN}-\mathrm{Stenotaphrum} \mathrm{secundatum} \mathrm{de} 3 \mathrm{~cm}$ de altura. Ambas evaluaciones fueron realizadas a las 8 de la mañana, en una temperatura ambiente entre 77-80 grados Fahrenheit y una humedad entre el 80-82\%.

\section{Análisis Estadístico}

Los datos obtenidos se digitaron en Excel y, para su posterior análisis, fueron exportados al software Stata 14,0 (StataCorp. 2015. Stata Statistical Software: Release 14. College Station, TX: StataCorp LP.). Las variables cuantitativas fueron presentadas en medianas y rangos intercuartílicos y las cualitativas, en frecuencias absolutas y relativas. El análisis de las diferencias de las variables espaciotemporales en superficie natural y artificial fue hecho mediante la prueba de rangos de Wilcoxon para datos pareados. De igual modo, se calculó el tamaño del efecto dividiendo el valor $\mathrm{Z}$ de la prueba rangos de Wilcoxon entre la raíz cuadrada del número de observaciones, que para este estudio corresponde a 32. El tamaño de efecto se interpretó así: $0.2=$ Pequeño; $0.5=$ Mediano; $0.8=$ Grande. Finalmente, las correlaciones entre las variables espaciotemporales fueron analizadas empleando el Coeficiente de Correlación de Spearman. Se consideró un nivel alpha del 5\% para todo el análisis.

\section{Resultados}

Las características de los participantes se muestran en la tabla 1. La mediana de edad fue de 12 años; la de talla 1,51 mts; la del peso $45 \mathrm{~kg}$ y la del Índice de Masa Corporal (IMC) $19,4 \mathrm{Kg} / \mathrm{m}^{2}$. La posición de juego para mayoría de jugadores fue mediocampista $(n=7)$, seguida por la línea defensiva $(n=6)$ y los delanteros $(n=5)$.

\begin{tabular}{|c|c|c|c|}
\hline & & Mediana & RIC \\
\hline \multicolumn{2}{|c|}{ Edad } & 12 & $(12-13)$ \\
\hline \multirow{3}{*}{$\begin{array}{c}\text { Variables } \\
\text { antropométricas }\end{array}$} & Talla (m) & 1.51 & $(1.48-1.60)$ \\
\hline & Peso (kg) & 45.0 & $(40.0-51.3)$ \\
\hline & $\operatorname{IMC}\left(\mathrm{kg} / \mathrm{m}^{2}\right)$ & 19.4 & $(17.4-20.8)$ \\
\hline Variable & & $\mathrm{N}=18$ & $\%$ \\
\hline \multirow{3}{*}{ Posición de juego } & Defensa & 6 & 33.3 \\
\hline & Mediocampista & 7 & 38.8 \\
\hline & Delantero & 5 & 28.7 \\
\hline
\end{tabular}

En la tabla 2, se muestra que la fase de apoyo de los futbolistas fue superior en SA $(p<0,05)$ con un tamaño de efecto mediano $(\mathrm{r}=0,621)$. A su vez, el Tv, la fase de contacto 
Tabla 2.

Variables espaciotemporales del patrón de carrera en superficie natural y artificial

Variable $\quad$ Superficie Natural $(\mathrm{n}=18)$ Superficie Artificial $(\mathrm{n}=18)$ Tamaño de Interpretación \begin{tabular}{cccccccc} 
& Mediana & RIC & Mediana & RIC & Valor $p$ & efecto $(\mathrm{r})$ & Del efecto \\
\cline { 1 - 6 } Velocidad $(\mathrm{m} / \mathrm{s})$ & 4.28 & $3.80 ; 5.36$ & 4.78 & $4.64 ; 4.96$ & 0.170 & 0.229 & Pequeño
\end{tabular} $\begin{array}{llllllll}\text { Aceleración }\left(\mathrm{m} / \mathrm{s}^{2}\right) & 0.40 & -0.26 ; 1.29 & 1.25 & 0.70 ; 1.91 & 0.058 & 0.316 & \text { Pequeño }\end{array}$

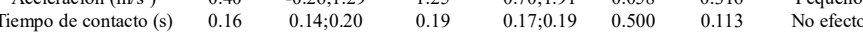
$\begin{array}{llllllll}\text { Tiempo de vuelo (s) } & 0.16 & 0.14 ; 0.19 & 0.04 & 0.04: 0.05 & <0.001 & 0.592 & \text { Mediano }\end{array}$ $\begin{array}{llllllll}\text { Fase de contacto }(\mathrm{s}) & 0.02 & 0.02 ; 0.03 & 0.01 & 0.01 ; 0.02 & 0.040 & 0.342 & \text { Pequeño }\end{array}$ Fase de Apoyo (s) $\quad \begin{array}{llllll}0.05 & 0.03 \cdot 0.06 & 0.09 & 0.08 \% 0.10 & <0.001 & 0.621\end{array}$ $\begin{array}{llllllll}\text { Fase de propulsión (s) } & 0.14 & 0.09 ; 0.17 & 0.07 & 0.06 ; 0.09 & <0.001 & 0.563 & \text { Mediano }\end{array}$ $\begin{array}{llllllll}\text { Zancada }(\mathrm{cm}) & 211 & 136 ; 238 & 199 & 193 ; 206 & 0.845 & 0.032 & \text { No efecto }\end{array}$

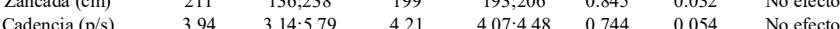
RIC: Rango Intercuartílico; $\mathrm{S}$ : segundos; m: metros; $\mathrm{p} / \mathrm{s}$ : pasos por segundo

y de propulsión fueron mayores en $\mathrm{SN}$ al ser comparada con la SA ( $\mathrm{p}<0,05)$, con tamaños de efecto mediano $(\mathrm{r}=0,592)$, pequeño $(0,342)$ y mediano $(0,563)$, respectivamente.

En la tabla 3, se observa una relación indirecta moderada entre la velocidad y la fase de contacto analizadas en la SN $(p<0,05)$. Adicionalmente, se observa una relación indirecta moderada entre la aceleración y el Tv, y una relación negativa moderada entre la aceleración y la zancada $(\mathrm{p}<0,05)$.

Tabla 3

Correlación entre variables espaciotemporales del patrón de carrera $(\mathrm{n}=18)$

\begin{tabular}{|c|c|c|c|c|}
\hline \multirow{2}{*}{ Variable } & \multicolumn{2}{|c|}{ Velocidad } & \multicolumn{2}{|c|}{ Aceleración } \\
\hline & SN & SA & SN & SA \\
\hline Tiempo de Contacto (s) & -0.23 & -0.31 & 0.29 & 0.41 \\
\hline Tiempo de Vuelo (s) & 0.12 & 0.02 & 0.43 & $-0.60^{*}$ \\
\hline Zancada $(\mathrm{cm})$ & -0.21 & 0.31 & -0.02 & $-0.50 *$ \\
\hline Cadencia $(\mathrm{p} / \mathrm{s})$ & 0.20 & 0.44 & -0.25 & 0.04 \\
\hline Fase de Contacto (s) & $-0.65^{*}$ & 0.08 & -0.13 & 0.31 \\
\hline Fase de Apoyo (s) & -0.45 & -0.18 & 0.02 & 0.15 \\
\hline Fase de propulsión (s) & 0.27 & -0.33 & -0.13 & -0.12 \\
\hline
\end{tabular}

\section{Discusión}

El análisis de los datos mostró que el patrón de carrera de los futbolistas analizados varía de acuerdo con la superficie utilizada. Así, se encontró que el Tv, la fase de contacto y de propulsión fueron superiores en la SA, y la fase de apoyo fue mayor en la SN. Por otra parte, los resultados obtenidos sugieren que el aumento de la velocidad en la SN puede estar determinado por una menor fase de contacto. Y que, en la SA, el aumento de la aceleración puede estar definitivo por un menor Tv y longitud de zancada.

En el presente estudio, la acción evaluada fue el sprint, el cual abarca entre el 0,5\% y el 5\% del tiempo total de juego; con respecto a la distancia, el sprint comprende del $1 \%$ al $12 \%$ del promedio recorrido por un futbolista durante el partido (Rienzi, Drust, Reilly, Carter, \& Martin, 2000; Gool, Gerven, Van \& Boutmans, 1988). Su importancia radica en que esta actividad se desenvuelve mediante recepciones, interceptaciones, pases, remates, conducción del balón y regates. Además, el sprint es considerado determinante en el éxito del juego si se compara con la distancia total recorrida en otro tipo de acciones. Algunos estudios indican que el sprint en línea recta sin oponente y sin balón, tal y como se evaluó en el presente estudio, es la acción desarrollada con mayor frecuencia antes del gol. (Andrzejewski, Chmura, Pluta, Strzelczyk, \& Kasprzak, 2013; Reilly, Bangsbo, \& Franks, 2000; FIFA, 2011).

Los hallazgos de velocidad y aceleración encontrados, mostraron ser mayores en SA con un tamaño de efecto pequeño; no obstante, esta diferencia entre superficies no fue significativa estadísticamente. Algunos estudios como los de (Nédélec et al, 2013; Gain et al, 2010; Choi et al, 2015), muestran velocidades y aceleraciones superiores al ser desarrolladas en SA. Lo anterior, es argumentado por Nédélec et al. (2013), quienes refieren que este tipo de superficie presenta mejores condiciones para realizar carreras de velocidad debido a una mayor fricción y absorción de impacto. Por su parte, Nagahara et al. (2018), así como Rabita et al (2018), demostraron que el incremento de las fuerzas propulsoras o de reacción con el suelo, en general, aumenta la aceleración. Adicionalmente, McMurtry \& Fiedler (2019), indican que la SA posee una mayor fuerza de reacción, por lo cual ésta superficie puede permitir el desarrollo de una mayor aceleración al ser comparada con la SN.

La uniformidad de las superficies sintéticas es otra de las posibles razones que puede promover el desarrollo de una mayor velocidad y aceleración. Así, esta condición disminuye el número de ajustes posturales que el futbolista debe realizar para compensar las irregularidades, más comunes, en las superficies de juego naturales (Pérez, Liana, Cortell \& Perez, 2009). Otros estudios afirman que no hay diferencias en la velocidad y aceleración desarrollada entre ambos tipos de superficies, siempre y cuando el pasto natural se encuentre en un estado óptimo (Gaudino, Gaudino, Alberti, \& Minetti, 2013; Jastrzêbski, Bichowska, Rompa, Radzimiñski, \& Dargiewicz, 2014; Diez \& Burillo, 2012; Andersson, Ekblom, \& Krustrup, 2008; Little \& Williams, 2005; Pauole, Madole, \& Lacourse, 2000; Baker \& Nance, 1999).

Si bien, la velocidad y la aceleración son indicadores del rendimiento en los futbolistas, también existen otras variables espaciotemporales estudiadas que pueden influir en las dos primeras. Según Rius (2005) y Murata et al (2018), las aceleraciones más altas se relacionan con un Tv más largo durante el sprint; no obstante, en el presente estudio esta variable no parece determinar la velocidad y en cambio, muestra una relación negativa con la aceleración alcanzada en SA.

Según Kampmiller, Vanderka, Šelinger, \& Šelingerová (2011), Rius (2005) y Rumpf, Cronin, Oliver, \& Hughes (2015), los parámetros que se deterioran parcialmente en la etapa prepuberal y puberal está el Tv, el cual se disminuye después de los 12.5 años, edad en la que se encontraba la mayor parte de los futbolistas evaluados. Estos mismos autores, así como Seagrave, Mounchbahani \& O‘donnel, 2009 y Mackala (2007), refieren que las variables principalmente relacionadas con la máxima velocidad en esta edad, son la longitud de zancada, el Tc y la cadencia, considerados como criterios determinantes para la selección de niños o jóvenes corredores.

En cuanto a la cadencia, los resultados del presente estudio no mostraron diferencias significativas entre ambas superficies, ni tampoco una asociación de ésta con la velocidad y la aceleración. Con relación a la longitud de zancada, los datos de este estudio evidenciaron una relación indirecta con la aceleración desarrollada en SA. Lo anterior, no está en consonancia con lo referido en la literatura consultada, en la cual el aumento de la longitud de zancada y la cadencia han sido considerados tradicionalmente como determinantes de la velocidad (Hunter, Marshall, \& McNair, 2004). Específicamente, Murphy et al., (2003) y Wild et al., (2018). consideran que de estas dos, la cadencia es la más influyente en el desarrollo de la velocidad en la fase inicial del sprint, etapa que fue analizada en la presente investigación.

Otra de las variables analizadas que mostró diferencias 
significativas entre superficies fue el tiempo de propulsión, el cual se mostró menor en la SA, donde hubo valores de velocidad y aceleración más altos, pero sin diferencia estadística. Según Paradisis, Bissas, Pappas, Zacharogiannis, Theodorou \& Girard (2019), un tiempo de propulsión más corto es una característica de los atletas más veloces, ya que les permite generar mayores fuerzas horizontales que se asocian de forma directa con la velocidad alcanzada principalmente en fases de aceleración temprana.

Finalmente, existieron variables que no pudieron ser estandarizadas como: la homogeneidad de la superficie que involucra las zonas de raíces, la zona de arena y la densidad de la grama. Así mismo, no fue posible controlar el tipo de botín, las fuerzas de absorción, las fuerzas de reacción entre las superficies de contacto, ni el patrón de sueño, como tampoco se controló la alimentación antes de las evaluaciones. Adicionalmente, es importante precisar que los jugadores de fútbol a menudo inician sus sprints desde una condición de movimiento previa y no estacionaria como se realizó en este estudio. Todo lo anterior pueden considerarse limitaciones que influyen en los resultados del patrón de carrera del futbolista juvenil y que deben ser tenidos en cuenta para estudios posteriores.

\section{Conclusión}

Los resultados obtenidos sugieren que la SA en los futbolistas juveniles evaluados permite un patrón de carrera con un menor tiempo de vuelo, una menor fase de contacto y de propulsión. Por otra parte, se encontró una asociación negativa entre la duración de la fase de contacto y la velocidad desarrollada en SN, asimismo, la duración del Tv y la zancada se asociaron negativamente con la aceleración en SA.

\section{Declaración de conflicto de interés}

No existen conflictos de interés alguno.

\section{Agradecimientos:}

Los autores agradecen al profesor Fausto Alarcón Quigua, al programa de fisioterapia de la UDES Bucaramanga y a la facultad de Cultura Física Deporte y Recreación de la USTA Bucaramanga, por su apoyo y acompañamiento a los grupos de semilleros Physis y SIAFS en la realización de la presente investigación.

\section{Referencias}

Blazevich, A. (2011). Biomecánica deportiva. Boldalona: Paidotribo.

Diez, O., \& Burillo, P. (2012). Influencia de la superficie de juego en el rendimiento de futbolistas amateurs: hierba natural, césped artificial y tierra. AGON International Journal of Sport Sciences, 2(2) 106-114.

Choi, S., Raymond, K., \& Elean, F. (2015). Comparison between Natural Turf and Artificial Turf on Agility Performance of Rugby Union Players. Advances in Physical Education, 5(4), 273-281.
Gaudino, P., Gaudino, C., Alberti, G., \& Minetti, A. (2013). Biomechanics and predicted energetics of sprinting on sand: Hints for soccer training. Journal of Science and Medicine in Sport, 16(3); 271-275.

González-De Los Reyes, Y.; Fernández-Ortega, J. \& GaravitoPeña, F. (2019) Características de fuerza y velocidad de ejecución en mujeres jóvenes futbolistas. Characteristics of Strength and Speed of Execution in Young Women Soccer Players. Revista Internacional de Medicina y Ciencias de la Actividad Física y el Deporte. 19(73); 167-179

Jastrzêbski, Z., Bichowska, M., Rompa, P., Radzimiñski, L., \& Dargiewicz, R. (2014). Influence of different types of surfaces on the results of running speed tests in young soccer players. Central European Journal of Sport Sciences and Medicine, 5(1), 5-14.

Faude O., Koch T. \& Meyer T. (2012). Straight sprinting is the most frequent action in goal situations in professional football. J. Sports Sci. 30 625-631.

Gain, G., Swedenhjelm, A., Mayhew, J., \& Houser, J. (2010). Comparison of speed and agility performance of college football players on field turf and natural grass. The Journal of Strength and Conditioning Research, 24(10), 2613-7.

Gool, V., Gerven, v., \& Boutmans. (1988). The physiological load imposed on soccer players during real match-play. In: Science and Football Committee. World Congress of Science and Football, Liverpool, Editorial Routledge.

Little, T., \& Williams, A. (2005). Speciûcity of acceleration, maximum speed and agility in professional soccer players. J Strength Cond Res, 19(1), 76-8.

Baker, D., \& Nance, S. (1999). he relation between running speed and measures of strength and power in professional rugby league players. J. Strength Cond. Res, 13(3), 224-229.

Pauole, K., Madole, K., Rozenek, R., \& Lacourse, M. (2000). Reliability and validity of the T-test as a measure of agility, leg power and leg speed in college aged men and women. J. Strength Cond. Res, 14; 450-443.

Rius, J. (2005). Metodología y técnicas de atletismo. Barcelona: Paidotribo.

Rumpf, M., Cronin, J., Oliver, J., \& Hughes, M. (2015). Kinematics and Kinetics of Maximum Running Speed in Youth Across Maturity. Pediatric Exercice Science, 27(2) 84-277.

Seagrave, L., Mouchbahani, R., \& O`donnel, K. (2009). Neurobiomechanics of maximum velocity sprinting. New Stud Athl, 24(1); 19-29.

Mackala, K. (2007). Optimisation of performance through kinematic analysis of the different phases of the 100 meters. New Stud Athl, 22(2); 7-16.

Avalos, J. (1-19 de 05 de 2017). Efectos del césped sintético y natural sobre el rendimiento físico y técnico en jugadores profesionales de fútbol. Obtenido de UNIVERSIDAD NACIONAL DE COSTA RICA: http:// repositorio.una.ac.cr/handle/11056/13654

Williams, J., Akogyrem, E., \& Williams, J. (2013). A MetaAnalysis of Soccer Injuries on Artificial Turf and Natural Grass. Journal of Sports Medicine, 1-6.

Sánchez-Sánchez, García-Unanue, Felipe, Jiménez-Reyes, Viejo-Romero, \& Gómez-López. (2016). Physical and physiological responses of amateur football players on 
third generation artificial turf systems during simulated game situations. J Strength Cond Res, 30(11); 77-3165.

Andersson, H., Ekblom, P., \& Krustrup, B. (2008). Elite football on artificial turf versus natural grass: Movement patterns, technical standards, and player impressions. Journal Sports Science, 26(2); 22-113.

Nédélec, M., McCall, A., Carling, C., Gall, L., Berthoin, F \& Dupont, G. (2013). Physical performance and subjective ratings after a soccer-specific exercise simulation: comparison of natural grass versus artificial turf. Journal Sports Science, 31(5); 36-529.

Rienzi, E., Drust, B., Reilly, T., Carter, J., \& Martin, A. (2000). Investigation of anthropometric and work-rate profiles of elite South American international soccer players. Journal Sport Sciene Physical Fitness, 40(2); 9-162.

Andrzejewski, M., Chmura, J., Pluta, B., Strzelczyk, R., \& Kasprzak, A. (2013). Analysis of sprinting activities of professional soccer players. J Strength Cond Res, 27(8); 40-2134.

Reilly, T., Bangsbo, J., \& Franks, A. (2000). Anthropometric and physiological predispositions for elite soccer. Journal Sports Science, 18(9); 83-669.

Optogait, M. (8 de 2018 de 2018). Optogait. Obtenido de Optogait: http://www.optogait.com/OptoGaitPortal/Media/Manuals/Manual-ES.PDF

Oyarzo-Mauricio, C. A., Said-Negrete, M. J., \& Nazar-Araya, M. J. (2017). Correlación del Single Hop Test con la prueba de Velocidad en treinta metros en infantes entre diez y doce años de un colegio privado de Santiago de Chile. RETOS. Nuevas Tendencias En Educación Física, Deporte y Recreación, 1(32), 101-105.

FIFA. (2011). Analisis del rendimiento físico durante la copa de la FIFA 2011. Zurich. Recuperado el 23 de Abril de 2016, de FIFA: https://resources.fifa.com/mm/document/ footballdevelopment/technicalsupport/01/68/06/99/ fwwcphysicalanalysis-ss.pdf.

Kampmiller, T., Vanderka, M., Šelinger, P., \& Šelingerová, M. (2011). Kinematic paramaters of the running stride in 7to 18-year-old youth. Kinesiol Slov, 17(2); 63-75.

Haugen, T. A., Tønnessen, E., Hisdal, J. \& Seiler, S. (2014). The role and development of sprinting speed in soccer. Int. J. Sports Physiol. Perform. 9, 432-441.

Murata, M., Takai, Y., Kanehisa, H., Fukunaga, T., \& Nagahara, R. (2018). Spatiotemporal and Kinetic Determinants of Sprint Acceleration Performance in Soccer Players. Sports (Basel, Switzerland), 6(4), 169.

Nagahara, R.; Mizutani, M.; Matsuo, A.; Kanehisa, H., \& Fukunaga, T. (2018) Association of sprint performance with ground reaction forces during acceleration and maximal speed phases in a single sprint. J.Appl. Biomech. $34,104-110$

Núñez J., Toscano, F., Suarez, L., Martinez I., \& Hoyos, M. (2019). Umbrales individualizados para analizar las demandas en la aceleración en futbolistas usando GPS. Retos, número 35; 75-79.

Rabita, G., Dorel, S., Slawinski, J., Saez-de-Villarreal, E., Couturier, A., \& Samozino, P.Morin, J.B. (2015) Sprint mechanics in world-class athletes: A new insight into the limits of human locomotion. Scand. J. Med. Sci. Sports , 25, 583-594.
Morin, J.B, Slawinski, J,Dorel, S., Saez-de-Villarreal, E., Couturier,A. Samozino, P, Brughelli, M. \& Rabita, G. (2015), Acceleration capability in elite sprinters and ground impulse: Push more, brake less? J. Biomech. 48, 3149-3154.

Murphy, A.J.; Lockie, R.G. \& Coutts, A.J. (2003) Kinematic determinants of early acceleration in field sport athletes. J. Sports Sci. Med. 2, 144-150.

Wild, J.J., Bezodis, I.N., North, J.S. \& Bezodis, N.E. (2018) Differences in step characteristics and linear kinematics between rugby players and sprinters during initial sprint acceleration. Eur. J. Sport Sci. 18(10); 1327-1337.

Paradicis, GP., Bissas, A., Pappas, P., Zacharogiannis, E., Theodorou, A., \& Girard, O. (2019). Sprint mechanical differences at maximal running speed: Effects of performance, J Sport Sci, 37(17); 2026-2036.

Pérez, P., Liana, S., Cortell, J., \& Perez, J. (2009) biomechanical factors to be taken into account to prevent injuries and improve sporting performance on artificial turf. J. Hum. Sport Exerc. 4(2); 78-92

Rabita G, Dorel S., Slawinski J., Sàez-de-Villarreal E., Couturier A., \& Samozino P. (2015). Sprint mechanics in world-class athletes: a new insight into the limits of human locomotion. Scand. J. Med. Sci. Sports. 25; 583-594.

McMurtry, S., \& Fiedler, G. (2019) Comparison of lower limb segment forces during running on artificial turf and natural grass. J Rehabil Assist Technol Eng. 6; 1-5.

Sánchez J., Hernández, C., Muñoz, V., González, A., Fernández, A., \& Carretero, M. (Efecto de un entrenamiento intermitente con y sin cambios de dirección, sobre el rendimiento físico de jóvenes futbolistas. Retos, número 30; 70-75.

Lee, M., Song, CH., Lee, KJ., Jung, SW., Shin, DC., \& Shin SH. (2014) Concurrent validity and test-retest reliability of the OPTOGait photoelectric cell system for the assessment of Spatio-temporal parameters of the gait of young adults. J Phys Ther Sci. 26(1); 81-85.

Lienhard, K., Schneider, D., \& Maffiuletti, NA. Validity of the Optogait photoelectric system for the assessment of spatiotemporal gait parameters. Med Eng Phys. 35(4); 4500.

Hunter, J.P., Marshall, R. N., \& McNair, P. J. (2004). Interaction of Step Length and Step Rate during Sprint Running. Medicine and Science in Sports and Exercise, 36(2), 261271.

Bezoidis, N., Willwacher, S., \& Tapio, A. (2019) The biomechanics of the track and fiel sprint start: A narrative Review. Sport Med. 49(9); 1345-1364.

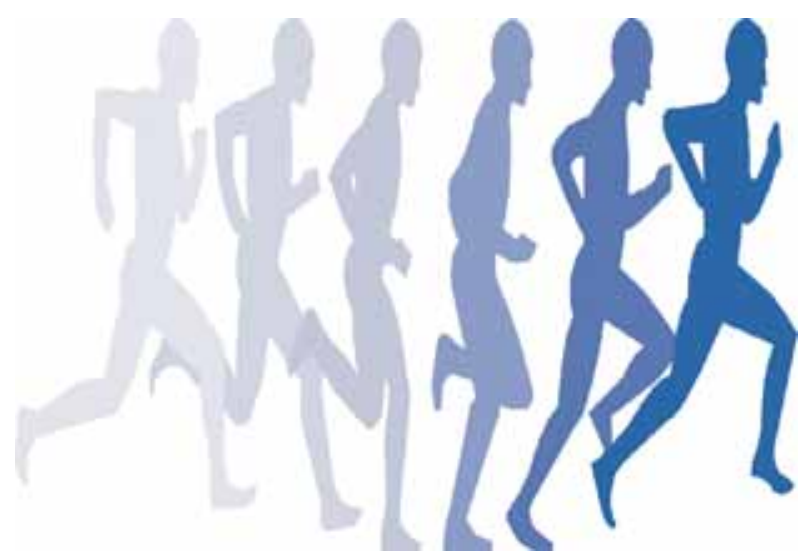

